\title{
Advanced experiment program on Fourier optics and digital color photography
}

Guoguang Mu, ZhiLiang Fang, Hongchen Zhai

Guoguang Mu, ZhiLiang Fang, Hongchen Zhai, "Advanced experiment program on Fourier optics and digital color photography," Proc. SPIE 4588, Seventh International Conference on Education and Training in Optics and Photonics, (28 May 2002); doi: 10.1117/12.468712

SPIE Event: Education and Training in Optics and Photonics 2001, 2001, Singapore, Singapore 


\title{
Advanced experiment program on Fourier optics and digital color photography
}

\author{
Guoguang Mu, Zhiliang Fang, and Hongchen Zhai \\ Institute of Modern Optics, Nankai University \\ Key Laboratory of Information Science and Technology, Ministry of Education, China \\ Weijing Road 94, Tianjin 300071, China
}

\begin{abstract}
We review the fundamental development in the last decade of white-light optical information processing for digital color photography and its instrumentation for the applications to education in white-light optical information processing. Applications to education in white-light information processing for graduated students majoring in physics, optics, information science, or opto-electronics will be introduced with emphasis.
\end{abstract}

Keywords: White-light, optical information processing, color photography, tri-color grating, digital camera

\section{OBJECTIVE IMAGING AND SPACE FREQUNCY SPECTRUM}

The roots of optical information processing can be traced back to Abbé's imaging theory in 1873 and Poter's experiment in 1906. They introduced for the first time the concept of spatial frequency spectrum and clearly analyzed the imaging principles of lenses with this Fourier theory. The inspiring effect of the spatial filter shown in the experiment was the most original contribution to the development in optical information processing at the mid-20th century.

Abbé's imaging theory can be introduced with the optical system shown in Fig. 1, where a monochromatic coherent collimated beam is diffracted by an object with a transmission function $f(x, y)$ in $P_{1}$, the front focus plane of $L_{1}$, the frequency spectrum $F(p, q)$ of the object $f(x, y)$ in $P_{2}$, the rear focal plane of $L_{1}$, can be then expressed as

$$
F(p, q)=\int_{-\infty}^{\infty} \int_{-\infty}^{\infty} f(x, y) e^{-i(p x+q y)} d x d y=\mathrm{F}[f(x, y)]
$$

where $p$ and $q$ are spatial frequency coordinates in the spectrum plane. As $P_{2}$ is just the front focus plane of $L_{2}$, the Fourier transform of $F(p, q)$ can be then obtained at plane $P_{3}$ as 


$$
f(-x,-y)=\int_{-\infty-\infty}^{\infty} \int^{\infty} F(p, q) e^{-i(p x+q y)} d p d q .
$$

In the case that $\alpha=-x, \beta=-y$ as showing in Fig.1, it can be rewritten as

$$
g(\alpha, \beta)=\int_{-\infty}^{\infty} \int_{-\infty}^{\infty} F(p, q) e^{i(p \alpha+q \beta)} d p d q=\mathrm{F}^{-1}[F(p, q)]
$$

It is noticed that $g(\alpha, \beta)$ is just an image of $f(x, y)$. Therefore, such an optical system executing a Fourier transform of a two-dimensional input object $\mathrm{f}(\mathrm{x}, \mathrm{y})$ and a successive reverse Fourier transform of its spectrum can be considered as an imaging system with computing function, in which $f(x, y)$ and $f(\alpha, \beta)$ is the input and output, respectively.

\section{COHERENT AND INCOHERENT OPTICAL IMAGING}

If a space filter $\mathrm{H}(\mathrm{p}, \mathrm{q})$ is inserted in $\mathrm{P}_{2}$ of the computing system above, the output in $\mathrm{P}_{3}$ will become

$$
g^{\prime}(\alpha, \beta)=\int_{-\infty-\infty}^{\infty} \int^{\infty} F(p, q) H(p, q) e^{i(p \alpha+q \beta)} d p d q
$$

Here, $g^{\prime}(\alpha, \beta)$ is obtained as an output of modified $g(\alpha, \beta)$, by applying a filter $H(p, q)$ on the frequency spectrum $F(p, q)$ of the input $f(x, y)$. Such a computing system can be understood as a coherent optical image processor shown in Fig. 2

In the early 1960s, such a kind of coherent optical image processors was widely used in many groups to improve qualities of images or to restore blurred images, among which the research work in Cutrona's group at Laboratory of Optics, Michigan University ${ }^{2}$ was the most representative example of the applications. They applied coherent optical processing techniques to synthetic-aperture radars successfully in drawing relief maps with high-resolution. Vander Lugt prepared spatial filters with coaxial holography ${ }^{3}$ and applied them successfully in optical correlation recognition or extracting signals from noisy background. To 1970s, coherent optical information processing was intensively studied and found widespread applications to the field such as analysis of optical spatial spectrum, deconvolution, image differential, addition and subtraction, synthesis of complex spatial filer, correlation recognition and so on. 
To overcome the difficulty that the processing quality of a coherent systems is usually restricted by the inevitable coherent noise or the quasi-monochromaticity of a coherent source, the research group in Electrical Department, Pennsylvania State University first proposed an incoherent white-light system for optical information processing in the late 1970s, as shown in Fig.3. This white-light processor is different from a typical coherent optical processor in the light source and the input plane, where an encoding plate and a Ronchi Grating is additionally inserted in front of the light source and in the input plane of the white-light processor, respectively. The former ensures a spatial coherency and the latter a spatial coherency, therefore, all the functions performed by a coherent optical processing system, such as complex spatial filtering, recovery of a blurred image, image addition or subtraction etc., can also be done successfully by this white-light information processor.

Suppose that a subject $s(x, y)$ in the input plane $P_{1}$ is close-contacted with a Ronchi grating of spatial frequency $p_{0}$, by illuminating by a collimated white-light beam, the spatial spectrum of the whole input in the plane $\mathrm{P}_{2}$ will be

$$
E\left(p, q, \lambda_{n}\right)=\iint s\left(x, y, \lambda_{n}\right) e^{-i P_{0} x} e^{-i(P x+q y)} d x d y=S\left(p-p_{0}, q, \lambda_{n}\right)
$$

where $S\left(p-p_{0}, q, \lambda_{n}\right)$ is the Fourier transform of $\mathrm{s}(\mathrm{x}, \mathrm{y})$ with a frequency shift $\mathrm{p}_{0}$ due to the modulation of the grating, and $\lambda_{n}$ denotes the wavelength included in the white light.

This spectrum will disperse along the axis $\alpha$ of the spatial frequency plane, resulting in a rainbow distribution. In the case that a spatial filter $\mathrm{H}$ with a narrow-band of wavelength $\alpha$

$$
H\left(p_{n}, q_{n}\right)=\left\{\begin{array}{cc}
\left(H\left(p_{n}, q_{n}\right)\right. & \alpha_{1}<\alpha<\alpha_{2} \\
0 & \text { otherwise }
\end{array}\right.
$$

is put at a correspondent position of $\mathrm{P}_{2}$, as shown in Fig.3, the output at wavelength $\lambda_{n}$ in $\mathrm{P}_{3}$ will become

$$
g\left(x, y, \lambda_{n}\right)=s\left(x, y ; \lambda_{n}\right) \times h\left(x, y ; \lambda_{n}\right) .
$$

For the spectra are incoherent to each other, the final intensity obtained in the output plane can be expressed as

$$
I(x, y)=\sum_{n} \Lambda_{n}\left|s\left(x, y, \lambda_{n}\right) \times h\left(x, y, \lambda_{n}\right)\right|^{2},
$$


which means that the output of this white-light processing system is an incoherent summation of the intensity over all the incoherent narrow-band spectra, as shown in Fig.4. Therefore, white-light information processing system has its advantages not only in avoiding coherent noise but also in the ability of multi-spectra information processing or wavelength division multiplexing (WDM). Thus, it has been successfully employed on color image processing, like color image addition and subtraction, pseudo-color encoding, archival storage of color films ${ }^{4}$ or color recovery of faded color films $s^{5}$, in which technique of white-light information processing expands the abilities and the application fields of a system of coherent light information processing.

\section{COLOR ENCODING AND DECODING IN BLACK-AND-WHITE FILMS}

\subsection{Color encoding and decoding in black-and-whit film}

Color encoding and color decoding are the basic techniques for recording color images in black-and-white films and for displaying the recorded color images, respectively, which can be used to store color images without color fading or to recover colors from color faded films, as detailed in refs. 4 and 5.

Fig. 5 shows the process for recording color information or faded color film onto a black-and-white film in time order. During the recording, a color transparency to be recorded is sandwiched between a Ronchi grating and a piece of black-and white positive film, and then, in turn with filters of different elementary color, red, green, and blue, respectively, the film is exposed to a white-light beam three times. After each exposure, the Ronchi grating is rotated around its normal into a new orientation. Thus, the information of the three elementary colors in the transparency is encoded onto the black-and-white film in a time series.

Fig.6 shows an optical decoding system used to display the color information recorded, where the encoded black-and-white positive film is placed in plane $P_{1}$ illuminated by a collimated beam of white light, and three filters of red, green, and blue elementary color are positioned at the correspondent first order of the spectrum in plane $\mathrm{P}_{2}$, respectively, while other area of the Fourier plane is opaque to the spatial spectrum. As a result, the restored color image will appear at the output plane.

Two images with faded colors and with recovered colors treated with the above technique are shown in Fig.7(a) and (b), respectively. It is noticed that the effect of color recovery is apparent, while the noise there is caused by what else.

\subsection{Color encoding with tri-color grating}

Based on the work above, we proposed a one-step recording method for color photography with black-and-white films 
in 1980s. ${ }^{6,7}$ By using a specially designed tri-color grating in this method, it is possible to encode color information on a black-and-white film with only one exposure, instead of the three described above. In the following, we discuss the basic principles of this one-step encoding as well as the decoding in more detail.

Fig. 8 is a scheme of the color encoding using a tri-color grating, where a photographic lens images a color object onto a black-and-white film with a close-contacted tri-color grating on its front side.

The construction of the tri-color grating is sketched in Fig.9. It is of three Ronchi gratings overlapping in different orientations, of which the transparent area is transparent to red, green and blue, respectively, so that the transmission of the tri-color grating can express as a sum of the three elementary gratings:

$$
T_{T}(x, y)=k\left\{\left[\frac{1}{2}+\frac{1}{2} \operatorname{sgn}\left(\cos p_{0} x\right)\right]_{R}+\left[\frac{1}{2}+\frac{1}{2} \operatorname{sgn}\left(\cos p_{0} x^{\prime}\right]_{G}+\left[\frac{1}{2}+\frac{1}{2} \operatorname{sgn}\left(\cos p_{0} x^{\prime}\right)\right]_{B}\right.\right.
$$

, where $\mathrm{p}_{0}$ is the grating frequency, and $\mathrm{x}, \mathrm{x}^{\prime}, \mathrm{x}^{\prime \prime}$ is the coordinate in the orientation of the three elementary grating, respectively.

For the amplitude of any color image of the camera can be expressed as the sum of the three elementary monochromatic images as

$$
T(x, y)=T_{r}(x, y)+T_{g}\left(x^{\prime}, y^{\prime}\right)+T_{b}\left(x^{\prime \prime}, y^{\prime \prime}\right)
$$

modulated by the grating $T_{T}(x, y)$, the intensity contribution of it on the film will be

$$
T_{P}(x, y)=K^{\prime}\left\{T_{r}(x, y)\left[\frac{1}{2}+\frac{1}{2} \operatorname{sgn}\left(\cos p_{0} x\right)\right]+T_{g}\left(x^{\prime}, y^{\prime}\right)\left[\frac{1}{2}+\frac{1}{2} \operatorname{sgn}\left(\cos p_{0} x^{\prime}\right)\right]+T_{b}\left(x^{\prime \prime}, y^{\prime \prime}\right)\left[\frac{1}{2}+\frac{1}{2} \operatorname{sgn}\left(\cos p_{0} x^{\prime \prime}\right)\right]\right\}^{\gamma} .
$$

If $\gamma$ can be controlled as 2 , the amplitude transmittance of the encoded black-and-white film will be

$$
\begin{aligned}
t_{p}(x, y)= & k^{\prime \prime}\left\{T_{r}(x, y)\left[\frac{1}{2}+\frac{1}{2} \operatorname{sgn}\left(\cos p_{0} x\right)\right]+T_{g}\left(x^{\prime}, y^{\prime}\right)\left[\frac{1}{2}+\frac{1}{2} \operatorname{sgn}\left(\cos p_{0} x^{\prime}\right)\right]\right. \\
& \left.+T_{b}\left(x^{\prime \prime}, y^{\prime \prime}\right)\left[\frac{1}{2}+\frac{1}{2} \operatorname{sgn}\left(\cos p_{0} x^{\prime \prime}\right)\right]\right\}
\end{aligned}
$$

\subsection{Optical decoding}


As described above, the color image encoded in the black-and-white film can be decoded in the $4 \mathrm{f}$ optical decoding system shown in Fig.6, of which the original color image will be displayed at the output plane $\mathrm{P}_{3}$. In the process, the encoded film with a transmission $t_{p}$ is placed in $P_{1}$, and the frequency spectrum of it will be

$$
\begin{aligned}
& E(p, q ; \lambda)=C \iint t_{P}(x, y) \exp [-i(p x+q y)] d x d y=T_{r}(\alpha, \beta)+\frac{1}{2} \sum_{n=1}^{\infty} a_{n} T_{r}\left(\alpha \pm \frac{n f \lambda}{2 \pi} P_{0}, \beta\right)+T_{g}\left(\alpha^{\prime}, \beta^{\prime}\right) \\
& +\frac{1}{2} \sum_{n=1}^{\infty} a_{n} T_{g}\left(\alpha^{\prime} \pm \frac{n f \lambda}{2 \pi} P_{0}, \beta^{\prime}\right)+T_{b}\left(\alpha^{\prime \prime}, \beta^{\prime \prime}\right)+\frac{1}{2} \sum_{n=1}^{\infty} a_{n} T_{b}\left(\alpha^{\prime \prime} \pm \frac{n f \lambda}{2 \pi} P_{0}, \beta^{\prime \prime}\right)
\end{aligned}
$$

With the three first orders $(n=1)$ filtered and the orders else than which shaded in the Fourier plane $P_{2}$, the inverse Fourier transform of the filtered spectrum will be output as an intensity contribution in $\mathrm{P}_{3}$

$$
I(x, y)=\left[T_{r}^{2}(x, y)\right]_{R}+\left[T_{g}^{2}\left(x^{\prime}, y^{\prime}\right)\right]_{G}+\left[T_{b}^{2}\left(x^{\prime \prime}, y^{\prime \prime}\right)\right]_{B}
$$

which is just the color image recorded.

Fig. 10 and Fig. 11 show two different optical systems designed for practical intrumentation.

\section{FOURIER OPTICS IN COLOR PHOTOGRAPHY}

In the last decade, we developed a series of devices, which can be applied to the color photography based on Fourier optics described above.

\subsection{Encoding cameras}

Figs. 13 and 14 are three different types of encoding cameras, BAZ-306 Vand BAZ-306® for $60 \mathrm{~mm}$ and $35 \mathrm{~mm}$ films, respectively, and BAZ-307 for areo-films of $190 \mathrm{~mm}$, of which each is a reassembled commercially available camera with a tricolor grating.

To fabricate the tri-gratings, the following technical key points should be taken into consideration: 1) color matching: specified white light could be synthesized by the light of red, green and blue transmitted through the grating; 2) appropriate spatial frequency, duty ratio and high contrast; 3) optimized orientations of the three elementary gratings to avoid the overlap of the spectrum and Morie fringes; 4) a precise design of mechanical parts of the grating, which should be close-contacted with the film during the photography and separated with the film afterwards automatically.

\subsection{Instrumentation for optical decoding}

The Figs.15 and 16 are two types of optical decoders matching BAZ-306 and BAZ-307, respectively, designed and 
fabricated in the Institute of Modern Optics, Nankai University, China. Based on the system shown in Figs.10 and 11 , the optical schemes are specially designed to meet all the requirements of color temperature, homogeneity of optical field, and elimination of different aberrations or stray light.

All these devices and instruments can be applied to the comprehensive courses of experimental optics related to Fourier Optics, such as image addition, subtraction, correlation, differential, convolution and so on.

\subsection{Computer-based digital decoding}

With more and more developments of the processing speed and performance in both hardware and software, for the decoding process, a PC computer-based digital system ${ }^{8,9}$ has obvious advantages over an optical system in flexibility of being connected to multimedia or transferred through network, as well as of the image size, though a parallel processing system would be generally expected in the most case of optical processing.

Fig12 shows a schematic drawing of digital decoding system, and Fig 17 is its computer system for $35 \mathrm{~mm}$ roll films. In the decoding process, the encoded film is digitalized and input to a PC computer by a scanner. The encoded image is then processed for Fourier transformation, color filtering, inverse Fourier transformation and image synthesized all in a computer, before the retrieved color image is output by a color printer or on a color monitor. By employing a new method of so-called Windowed Fourier Transform, the processing time for decoding an encoded film of size $180 \mathrm{~mm} \times 180 \mathrm{~mm}$ has been reduced from 3 hours to 3 minutes.

It is convenient also for a computer-based digital decoding system to employing a fusion technique of zero order spectrum to improve the resolution of a color image. Besides, the nonlinear errors arose from the encoding or the chemical processes in the black-and-white films can be corrected by a color correction matrix in the computer system, which further more improves the quality of the color image.

\section{ARCHIVAL STORAGE OF COLOR FILMS}

Color encoding and decoding techniques open up the possibility for long term archival storage of color films without any color fading. Fig 18 shows the pictures taken by BAZ-306 $83 \mathrm{kms}$ away from the scene, of which Fig. 18a is the encoded film and Fig. $18 \mathrm{~b}$ is the decoded color image. It is noticed that it would be almost impossible to reach such a color saturation using conventional photographic technique, if the picture is taken so far away from the scene. Fig. 19a shows an encoded black-and white image of lotus taken from a near distance by the encoding camera BAZ-306 Vten

years ago. Thanks to the encoding technique in black-and-white film, the fresh color of the lotuses in the decode image of Fig. 19b shows successfully the ability of the technique for a long-term archival storage of color films. Figs.20a and $20 \mathrm{~b}$ are the same kind of example for a long-term archival storage of color films, except that the encoding camera BAZ-306 ${ }^{\circledR}$ is used in this case. Figs. $21 \mathrm{a}$ and $21 \mathrm{~b}$ show the result of encoding camera BAZ-307 recording a topographic scene at a high altitude. Again, it is possible to display the photogram with a high color saturation, while otherwise the 
color saturation would be much lower by conventional photography.

\section{FURTHER IMPROVEMENTS ON DIGITAL CAMERAS}

\subsection{Construction of the color pixels}

Fig. 22(a) shows the contributions of the tri-color sensitive nerve cells of a kind of primate animals' retina. It is noticed that human being's retina with three different kinds of photosensitive nerve cells to different spectra band is in a way similar to this distribution, where the different three kinds of cells are symmetric in neither their spatial distribution or their amount. Comparing the distribution difference of their photo-sensitive pixels in the three figures, Figs. 22(a) to 22(c), we believe that it would be possible to optimize the tri-color grating or the Bayer filter of the conventional digital camera in a way that the identity of the amount and the spatial frequency of the three colors may not be necessary at all.

\subsection{Fusion of images}

Figs. $23 \mathrm{a}$ and $23 \mathrm{~b}$ show the results of the decoded color images, with and without the zero order spectrum of the encoded black-and -white image, respectively. It is noticed that the fusion of the image decoded from the zero order spectrum with that from the three first order spectra is an effective method to improve the quality of the decoded color image.

\section{CONCLUSIONS}

In this paper, we have introduced a new technique and its instrumentation of white-light information processing applied to the color photography, which is of the following advantages:

(1) Black-and-white films can be used to record color images by encode the color information in different orientations with a specially designed tri-color grating. During the decoding, the color filtering in the frequency plane can be used to retrieve all the color information, without the chemical process of development and fixation. This technique is advantageous to avoid fading of the color information in its storage.

(2) Different from the three-sequential-exposure encoding method with three different color gratings, our single-exposure encoding method using the tri-color grating makes it possible to make color photography with black-and-white films in real-time, of which the encoded and the decoded color images taken by the three different types of encoding cameras of BAZ-306, , BAZ-306® and BAZ-307, are presented in Figs. 18 to 21 , respectively.

( 3 ) In the process of encoding, the information included in a color image, expressed by $f(x, y)_{R}, f(x, y)_{G}$, and $f(x, y)_{B}$, is compressed into a two-dimensional distributed intensity function, $\mathrm{I}(\mathrm{x}, \mathrm{y})$, which is advantageous in digital information communications. 
(4) The decoding can be processed either by a $4 \mathrm{f}$ white-light information processing system or by a computer-based digital system, and the later is easy to be connected with networks for practical applications.

(5) The color scenes taken by the encoding cameras away from a long distance can be displayed in high color saturation, while that taken by a conventional camera will not, because of absorptions or dispersions in the air.

( 6 ) Color information encoded in black-and-white films can be stored for a long time without any fading, and it can be, therefore, consider as an alternative method for color archive storage other than that using magnetic discs or CDs.

( 7 ) The principle of encoding color information in black-and-white films can be applied in generally to any kind of recording systems with color-blind photo-sensors to design a new type of digital color camera.

( 8 ) White-light information processing applied to color photography can be implemented as a system for advanced experiment of modern optics.

\section{ACKNOWLEDGEMENTS}

The work presented here is based on the collaboration with Institute of Modern Optics, Nankai University, Key Laboratory of Information Science and Technology, Ministry of Education, China, Changchun Institute of Optics and Fine Mechanics, Physics Institute of Remote Sensing of Beijing, Nanjing Factory 5311 \& 3304, and Phenix Optical Instrument Factory.

\section{REFERENCES}

1. Guoguang Mu et al., Optics, The People's Education Press , PP. 379-400. 1978

2. Cutrona L.J. "On the application of coherent optical processing techniques to systhetic-apetine radar," Proc IEEE, PP. 54-1026, 1965

3. Vander lugt A.B. "Signal Detection by complex Spatial filtering," IEEE Trans. Inform Theory, IT-10, pp. 2-6, 1964

4. Frausis T.S , “White-Light Processing Technique for Archival Storage of color films, ” Appl. Opt., 19. pp. 457-459, 1980

5. Yu F.T.S and Mu G.G. etc., “ Color Restoration of faded color films, ” Optik, 58, pp. 389-340, 1981

6. Guoguang $\mathrm{Mu}$, et al., "A white-light processing technique for color grating", Chinese Journal of Scientific Instruments, 4, pp. 125-130, 1983

7. G.G. Mu et al., "Color photography with black-and-white film," Chinese Patent, CN1003811B, E28, 1989.

8. Mu G.G. etc. "Color data image encoding method and apparatus with spectral zone-filter," US patent, 5452002 1995-09 
9. Mu G.G. etc. "A physical method for color photography" Trends in Optics ICOIII, ed. Consoctini A, New York, Academic press, pp. 527-542, 1996

10. G.Luo, F.L. Liu, L. Lin, et al., "The technique of optical/ digital color photography based on white-light information processing," Science in China (Series E), 30, pp. 223-229, 2000 